Article

\title{
The Nitrogen Stress-Repressed sRNA NsrR1 Regulates Expression of all1871, a Gene Required for Diazotrophic Growth in Nostoc sp. PCC 7120
}

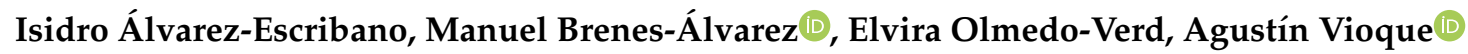 \\ and Alicia M. Muro-Pastor *(1) \\ Instituto de Bioquímica Vegetal y Fotosíntesis, Consejo Superior de Investigaciones Científicas and Universidad \\ de Sevilla, 41092 Sevilla, Spain; isidroae9@hotmail.com (I.Á.-E.); mabrea192@gmail.com (M.B.-Á.); \\ eolmedoverd@hotmail.com (E.O.-V.); vioque@us.es (A.V.) \\ * Correspondence: alicia@ibvf.csic.es; Tel.: +34-954489521
}

Received: 25 March 2020; Accepted: 27 April 2020; Published: 29 April 2020

check for updates

\begin{abstract}
Small regulatory RNAs (sRNAs) are post-transcriptional regulators of bacterial gene expression. In cyanobacteria, the responses to nitrogen availability, that are mostly controlled at the transcriptional level by NtcA, involve also at least two small RNAs, namely NsiR4 (nitrogen stress-induced RNA 4) and NsrR1 (nitrogen stress-repressed RNA 1). Prediction of possible mRNA targets regulated by NsrR1 in Nostoc sp. PCC 7120 allowed, in addition to previously described $n b l A$, the identification of all1871, a nitrogen-regulated gene encoding a protein of unknown function that we describe here as required for growth at the expense of atmospheric nitrogen $\left(\mathrm{N}_{2}\right)$. We show that transcription of all1871 is induced upon nitrogen step-down independently of NtcA. All1871 accumulation is repressed by NsrR1 and its expression is stronger in heterocysts, specialized cells devoted to $\mathrm{N}_{2}$ fixation. We demonstrate specific interaction between NsrR1 and the $5^{\prime}$ untranslated region (UTR) of the all1871 mRNA, that leads to decreased expression of all1871. Because transcription of NsrR1 is partially repressed by NtcA, post-transcriptional regulation by NsrR1 would constitute an indirect way of NtcA-mediated regulation of all1871.
\end{abstract}

Keywords: regulatory RNA; cyanobacteria; post-transcriptional regulation; heterocyst

\section{Introduction}

Small non-coding RNAs (sRNAs) are relevant players in regulatory circuits affecting essentially every aspect of bacterial physiology. These types of molecules are usually post-transcriptional regulators fine-tuning the responses to different environmental conditions [1]. In fact, the interplay between the regulation exerted by transcription factors and that exerted by small RNAs produces complex regulatory circuits in the form of feed-forward loops (coherent or incoherent) involving a transcription factor, an sRNA and their regulated target(s) [2,3].

Global nitrogen regulation is controlled in cyanobacteria by NtcA, a protein that belongs to the CRP/FNR family of transcriptional regulators [4]. Direct binding of NtcA to the corresponding promoters accounts for regulation of many nitrogen-regulated genes [5-7]. However, the mechanisms involved in expression of some nitrogen-regulated genes whose promoters do not contain NtcA binding sites might involve the participation of other NtcA-regulated factor(s). NtcA has been shown to regulate expression of several sRNAs, some of them with a wide distribution among phylogenetically distant cyanobacteria $[5,8,9]$. Among these sRNAs, NsiR4 (nitrogen-stress inducible RNA 4) is involved in nitrogen assimilation control via regulation of IF7, the inactivating factor of the key enzyme glutamine synthetase [8]. NsrR1 (nitrogen-stress repressed RNA 1) modulates translation of NblA [10], a protein 
required for the degradation of phycobilisomes that provide amino acids as a source of nitrogen under nitrogen deficiency [11]. Transcription of both gif $A$ (encoding IF7) and $n b l A$ is directly regulated by NtcA [5,12], therefore the post-transcriptional regulation exerted on these transcripts by NsiR4 and NsrR1, respectively, constitutes a second level of NtcA-mediated, indirect regulation.

In the absence of combined nitrogen, filamentous cyanobacteria such as model strain Nostoc sp. PCC 7120 differentiate heterocysts, specialized cells devoted to fixation of atmospheric nitrogen $[13,14]$. Differentiation of functional heterocysts is ultimately under control of NtcA, but also requires HetR, a regulator specifically involved in cellular differentiation. The nitrogen-regulated, HetR-dependent transcriptome includes transcripts for genes involved in specific aspects of heterocyst physiology, such as the sequential deposition of specialized envelopes or the fixation of nitrogen by the enzyme nitrogenase. The HetR-dependent transcriptome also includes non-coding transcripts, both antisense and small RNAs, that would participate in the metabolic reprogramming that takes place in heterocysts $[15,16]$, again pointing to the relevance of post-transcriptional regulation on cyanobacterial physiology.

In this work, we identify all1871 as a gene required for heterocyst function and describe its regulation by NsrR1. Expression of all1871 is induced upon nitrogen step down, but its induction does not require NtcA or HetR. We verify that NsrR1 regulates accumulation of All1871 at the post-transcriptional level by its interaction with the $5^{\prime}$-UTR of all1871.

\section{Materials and Methods}

\subsection{Strains and Growth Conditions}

Cultures of wild-type and the different mutant derivatives of Nostoc sp. PCC 7120 (Table S1) were bubbled with an air/ $\mathrm{CO}_{2}$ mixture $(1 \% v / v)$ and grown photoautotrophically at $30{ }^{\circ} \mathrm{C}$ in BG11 medium [17] containing ferric citrate instead of ammonium ferric citrate, lacking $\mathrm{NaNO}_{3}$ and containing $6 \mathrm{mM} \mathrm{NH}_{4} \mathrm{Cl}, 10 \mathrm{mM} \mathrm{NaHCO}_{3}$, and $12 \mathrm{mM} \mathrm{N}$-tris(hydroxymethyl)methyl-2-aminoethanesulfonic acid-NaOH (TES) buffer (pH 7.5). Nitrogen deficiency was induced by removal of combined nitrogen. Occasionally, $17.6 \mathrm{mM} \mathrm{NaNO}_{3}$ was used as nitrogen source. Solid media were solidified with $1 \%$ Difco Agar. Mutant strains were grown in the presence of appropriate antibiotics at the following concentrations: streptomycin (Sm) and spectinomycin (Sp), 2-3 $\mu \mathrm{g} / \mathrm{mL}$ each (liquid medium) or $5 \mu \mathrm{g} / \mathrm{mL}$ each (solid medium), neomycin (Nm), $5 \mu \mathrm{g} / \mathrm{mL}$ (liquid medium) or $25 \mu \mathrm{g} / \mathrm{mL}$ (solid medium). Escherichia coli strains (Table S1) were grown in Luria-Bertani (LB) medium, supplemented with appropriate antibiotics.

\subsection{Reporter Assays for In Vivo Verification of Targets}

For the experimental target verification in E. coli, we used a previously described reporter system [18] and the superfolder green fluorescent protein (sfGFP) plasmid pXG10-SF [19]. The 5'-UTR of all1871, from the transcriptional start site (TSS) at position -137 with respect to the initiation codon (coordinate 2234072) to 60 nucleotides within the coding region, containing the predicted NsrR1 interaction sequence, was amplified from genomic DNA using oligonucleotides 247 and 248 (see Table S2 for oligonucleotide sequences and description). The information about the TSS was taken from [5]. The corresponding polymerase chain reaction (PCR) product was digested with NsiI and XbaI and cloned into the vector pXG10-SF digested with NsiI and NheI, resulting in plasmid pIAE9, bearing a translational fusion of a truncated All1871 protein with sfGFP (see Table S3 for plasmid descriptions). For NsrR1 expression in E. coli, plasmid pAVN1 [10] was used (Table S3).

Mutation U51G (Mut-51) was introduced in NsrR1 by overlapping PCR using primer pairs 197 and 296, and 198 and 295, and cloned as described for the wild type version [10] generating plasmid pIAE20 (Table S3). The compensatory mutation in the 5'-UTR of all1871 (Comp-51) was generated in the same way with primer pairs 247 and 304, and 303 and 248, and cloned as described above for the wild-type version resulting in plasmid pIAE22 (Table S3). The sequences of inserts in plasmids containing NsrR1 and all1871-sfgfp fusions are shown in Tables S4 and S5, respectively. 
For testing various combinations of both plasmids, these were introduced into E. coli DH5 $\alpha$. Plasmid pJV300 [20] was used as a control expressing an unrelated RNA. Plasmid pXG0 [18] was used as control for background fluorescence. Fluorescence measurements were done with a microplate reader (Varioskan) using liquid cultures from eight individual colonies bearing each combination of plasmids, and normalized to the $\mathrm{OD}_{600}$ of each culture as described previously [21]. Fluorescence was also visualized in $E$. coli cells plated on solid LB medium by excitation with a 302-nm wavelength lamp.

\subsection{RNA Isolation, Northern Blot and Primer Extension Analysis}

RNA samples were isolated from cells collected at different times after removing combined nitrogen (ammonium) from the media. Alternatively, cells were grown in media lacking combined nitrogen and RNA was isolated from cells collected at different times after the addition of $10 \mathrm{mM} \mathrm{NH}_{4} \mathrm{Cl}$ and $20 \mathrm{mM}$ TES buffer. Total RNA was isolated using hot phenol as described [22] with modifications [9]. Northern blot hybridization was performed as previously described [23,24]. Strand-specific ${ }^{32} \mathrm{P}$-labelled probes for Northern blot were prepared with Taq DNA polymerase using a PCR fragment as template in a reaction with $\left[\alpha-{ }^{32} \mathrm{P}\right] \mathrm{dCTP}$ and one single oligonucleotide as primer (corresponding to the complementary strand of the sRNA or mRNA to be detected). Hybridization to rnpB [25] was used as loading and transfer control. Hybridization signals were quantified on a Cyclone Storage Phosphor System with Optiquant software (PerkinElmer). Primer extension analysis of 5' ends of all1871 was carried out as previously described [23] using $5 \mu \mathrm{g}$ of total RNA and oligonucleotide 161 labeled with $\left[\gamma_{-}{ }^{32} \mathrm{P}\right]$ ATP.

\subsection{In Vitro Synthesis and Labelling of RNA}

The DNA templates for the in vitro transcription of NsrR1 and all1871 5'-UTR RNA were generated by PCR with a forward primer that includes a T7 promoter sequence and three extra Gs upstream the $5^{\prime}$-end of the coded RNA, and a reverse primer corresponding to the $3^{\prime}$ end of the RNA (see Tables S2 and S6). The all1871 5'-UTR fragment extends from the TSS at position -137 to 60 nucleotides downstream the translational start. RNA transcripts were generated with the MEGAscript High-Yield Transcription Kit (AM1333, Ambion). After transcription, RNAs were treated with DNase I and purified by phenol and chloroform extraction, ethanol-precipitated at $-20{ }^{\circ} \mathrm{C}$, and washed with $70 \%$ ethanol. In vitro transcribed RNAs were $5^{\prime}$-labelled and purified as described [10].

\subsection{In Vitro Structure Probing and Footprinting}

We mixed 0.1 pmol (about 50,000 cpm) of labeled NsrR1 RNA in $7 \mu \mathrm{L}$ with 2 pmol of unlabelled all1871 5'-UTR RNA, denatured for $1 \mathrm{~min}$ at $95{ }^{\circ} \mathrm{C}$ and chilled on ice for $5 \mathrm{~min}$, followed by the addition of $1 \mu \mathrm{L}$ of $1 \mathrm{mg} / \mathrm{mL}$ yeast RNA (Ambion AM7118) and $1 \mu \mathrm{L}$ of $10 \times$ structure buffer (Ambion). The samples were incubated further for $15 \mathrm{~min}$ at $37^{\circ} \mathrm{C}$. Treatment with RNase T1, RNase A or lead(II) acetate were performed as described [10].

An alkaline ladder was obtained by incubating 0.2 pmol of $5^{\prime}$-labelled RNA at $95^{\circ} \mathrm{C}$ for 3 min in $7.5 \mu \mathrm{L}$ of alkaline hydrolysis buffer (Ambion) containing $1.5 \mu \mathrm{g}$ of yeast RNA (Ambion AM7118). Reactions were stopped by the addition of $15 \mu \mathrm{L}$ of denaturing formamide loading buffer.

RNase T1 G ladders were obtained by incubating 0.1 pmol of $5^{\prime}$-labelled RNA and $1 \mu \mathrm{L}$ of $1 \mathrm{mg} / \mathrm{mL}$ yeast RNA (Ambion AM7118) in $9 \mu \mathrm{L}$ sequencing buffer (Ambion) for $10 \mathrm{~min}$ at $50{ }^{\circ} \mathrm{C}$, followed by the addition of $1 \mu \mathrm{L}$ of $0.1 \mathrm{U} / \mathrm{mL}$ RNase T1 (Ambion AM2283) and incubation at room temperature for $15 \mathrm{~min}$. Reactions were stopped by the addition of $20 \mu \mathrm{L}$ of Inactivation/Precipitation buffer (Ambion) and incubation at $-20{ }^{\circ} \mathrm{C}$ for $15 \mathrm{~min}$. The precipitate was washed with $70 \%$ ethanol and resuspended in 3-7 $\mu \mathrm{L}$ of denaturing formamide loading buffer.

All samples were run on 10\% polyacrylamide, $7 \mathrm{M}$ urea gels and bands visualized with a Cyclone Storage Phosphor System (PerkinElmer). 


\subsection{Expression and Purification of Protein All1871 and Western Blot}

To produce His-tagged All1871 protein, the all1871 gene was amplified using Nostoc DNA as template and primers 335 and 336, and the PCR product was cloned in vector pET-28a (+) (Novagen) using NcoI and XhoI, producing plasmid pIAE30. Plasmid pIAE30, containing downstream a T7 polymerase-dependent promoter the all1871 gene fused at the $3^{\prime}$ end to a sequence encoding a $\mathrm{His}_{6}$-tag, was transferred by electroporation to E. coli BL21-(DE3)-RIL, in which the gene encoding T7 RNA polymerase is under the control of an isopropyl- $\beta$-D-1-thiogalactopyranoside (IPTG)-regulated promoter. A $25-\mathrm{mL}$ pre-inoculum of this strain was grown overnight in LB medium supplemented with chloramphenicol and kanamycin and used to inoculate $275 \mathrm{~mL}$ of the same medium. The culture was incubated at $37^{\circ} \mathrm{C}$ until $\mathrm{OD}_{600}=0.6$. Recombinant All1871 expression was induced by the addition of $1 \mathrm{mM}$ IPTG. After $4 \mathrm{~h}$ at $37^{\circ} \mathrm{C}$, cells were collected and resuspended in $20 \mathrm{mM}$ sodium phosphate buffer ( $\mathrm{pH} 7.2$ ) containing $6 \mathrm{M}$ urea, $500 \mathrm{mM} \mathrm{NaCl}, 5 \mathrm{mM}$ imidazole and $1 \mathrm{mM}$ phenylmethylsulfonyl fluoride $(6 \mathrm{~mL} / \mathrm{g}$ of cells). Cells were broken by sonication and after centrifugation at $15,000 \times g(15 \mathrm{~min}$, $4{ }^{\circ} \mathrm{C}$ ), and the $\mathrm{His}_{6}$-All1871 protein was purified from the supernatant by chromatography through a 1-mL HisTrap HP column (GE Healthcare), using an imidazole gradient in the same buffer to elute the retained proteins. An additional purification step was carried out by size-exclusion chromatography in a HiLoad 16/60 Superdex 75 column (Pharmacia) using $20 \mathrm{mM}$ sodium phosphate buffer (pH 7.2) containing $2 \mathrm{M}$ urea and $150 \mathrm{mM} \mathrm{NaCl}$. A total amount of $3.5 \mathrm{mg}$ of purified protein was used in seven subcutaneous injections of a rabbit to produce antibodies in the Animal Production and Experimentation Center, Universidad de Sevilla (Seville, Spain). Antiserum was recovered several times up to five months after the first injection and stored at $-80^{\circ} \mathrm{C}$ until used. Antibodies specific for All1871 were purified from the serum by affinity chromatography on immobilized His-tagged All1871 using the AminoLink ${ }^{\circledR}$ Plus Immobilization Kit (ThermoFisher, Waltham, MA, USA) following the manufacturer's instructions.

For Western blot analysis, E. coli cells were resuspended in sodium dodecyl sulphatepolyacrylamide gel electrophoresis (SDS-PAGE) loading buffer and the proteins fractionated on 15\% SDS-PAGE. Antibodies against All1871 (see above), GFP (Roche) and E. coli GroEL (SIGMA-ALDRICH) were used. The ECL Plus immunoblotting system (GE Healthcare) was used to detect the different antibodies using anti-rabbit (SIGMA-ALDRICH) or anti-mouse (Bio-Rad) horse radish peroxidase conjugated secondary antibodies.

For Western blot analysis of Nostoc proteins, soluble fractions of cell-free extracts were used. Cells from $25 \mathrm{~mL}$ of culture were resuspended in $500 \mu \mathrm{L}$ of Tris- $\mathrm{HCl}$ buffer ( $\mathrm{pH}$ 8) containing $2 \mathrm{mM}$ $\beta$-mercaptoethanol and protease inhibitor cocktail (cOmplete ${ }^{\mathrm{TM}}$ ultra tablets, EDTA-free, Roche) in an Eppendorf $1.5 \mathrm{~mL}$ tube. A volume of approximately $75 \mu \mathrm{L}$ of glass beads $(0.25-0.3 \mathrm{~mm}$ diameter) was added and the suspension subjected to seven cycles of $1 \mathrm{~min}$ vortex followed by $1 \mathrm{~min}$ on ice. The resulting extract was centrifuged $3 \mathrm{~min}$ at $3000 \times g$ at $4{ }^{\circ} \mathrm{C}$ and the supernatant further centrifuged for $30 \mathrm{~min}$ at $16,000 \times \mathrm{g}$ at $4{ }^{\circ} \mathrm{C}$. The supernatant of the last centrifugation constitutes the soluble fraction.

\subsection{Generation and Complementation of all1871 Mutant Strain}

To generate a strain lacking all1871, two overlapping fragments were amplified by PCR using as template genomic DNA with oligonucleotides 162 and 163 and oligonucleotides 164 and 165, respectively (Table S2). The resulting products were then used as templates for a third PCR with oligonucleotides 162 and 165 resulting in deletion of sequences corresponding to all1871 and the generation of a unique XhoI site between the two amplified fragments. The fragment was cloned into pSpark (Canvax Biotech), rendering pSAM318 and its sequence was verified (Eurofins Genomics). After digestion with BamHI at the sites provided by oligonucleotides 162 and 165, the fragment was cloned into BamHI-digested $s a c B$-containing $\mathrm{Sm}^{\mathrm{R}} \mathrm{Sp}^{\mathrm{R}}$ vector $\mathrm{pCSRO}$ [26], rendering pSAM324. A Nm${ }^{R}$ gene was excised from pRL278 [27] as a Sall-XhoI fragment and cloned into the Xhol site in pSAM324, rendering pSAM326, which was transferred to Nostoc sp. strain PCC 7120 by conjugation [28], with selection for resistance to $\mathrm{Nm}$. Cultures of the exconjugants obtained were used to select for 
clones resistant to 5\% sucrose [29], and individual sucrose resistant colonies were checked by PCR using flanking oligonucleotides 162 and 180. Clones bearing the all1871 region interrupted by the $\mathrm{Nm}^{\mathrm{R}}$ gene were named all1871::Nm.

Plasmid pIAE65 was constructed to express all1871 from the trc promoter. A PCR fragment was amplified using oligonucleotides 247 and 735, digested with NsiI and XhoI and cloned into NsiI and XhoI-digested pMBA37 [15]. pIAE65 was transferred to strain all1871::Nm by conjugation with selection of $\mathrm{Sm}^{\mathrm{R}} \mathrm{Sp}^{\mathrm{R}}$ cells.

\subsection{Construction of a Strain Bearing a Translational Fusion all1871-gfpmut2}

In order to analyze spatial expression of all1871 along Nostoc filaments, plasmid pELV75 (Table S3) was constructed. A fragment containing the sequence from position -200 with respect to the TSS plus the entire all1871 gene was amplified using oligonucleotides 451 and 596. The fragment was digested with ClaI and EcoRV and cloned in pCSAM147 [30], in frame with the gpfmut2 gene, rendering pELV73. The EcoRI fragment from pELV73 containing the all1871-gfpmut2 translational fusion was cloned into pCSV3 [31] producing pELV75, that was transferred to Nostoc by conjugation with selection for $\mathrm{Sm}^{\mathrm{R}} \mathrm{Sp}^{\mathrm{R}}$ cells.

\subsection{Microscopy}

Fluorescence of Nostoc sp. PCC 7120 filaments carrying plasmid pELV75 growing on top of solidified nitrogen-free medium, was analyzed by confocal microscopy and quantified as described [32] using a Leica HCX PLAN-APO 63× 1.4 NA oil immersion objective attached to a Leica TCS SP2 laser-scanning confocal microscope. Samples were excited at $488 \mathrm{~nm}$ by an argon ion laser and the fluorescent emission was monitored by collection across windows of 500-538 nm (GFP) and 630-700 nm (cyanobacterial autofluorescence). Filaments were stained with Alcian blue and visualized at the microscope as described [33].

\section{Results}

\subsection{NsrR1 Interacts with all1871 mRNA $5^{\prime}-U T R$}

NsrR1 (nitrogen stress-repressed RNA 1) is a small RNA previously described to post-transcriptionally regulate expression of $n b l A$ [10]. A prediction of mRNAs possibly regulated by NsrR1 also identified the $5^{\prime}$-UTR of gene all1871 as likely interacting with NsrR1 [10]. all1871 would encode a conserved protein of unknown function. The high score of the prediction $\left(p\right.$-value $\left.=6.11 \times 10^{-9}\right)$, together with the conservation of predicted interactions between NsrR1 homologs and all1871 homologs in most cyanobacteria encoding NsrR1 (Figure S1), prompted us to further analyze a possible post-transcriptional regulation of all1871 by NsrR1.

Figure 1 shows the predicted interaction between NsrR1 from Nostoc sp. PCC 7120 and the $5^{\prime}$-UTR of all1871, that extends from position -40 to -3 , overlaps the translation initiation region and therefore is expected to affect translation of the mRNA (Figure 1A). To verify the interaction between NsrR1 and the mRNA of all1871, we used a heterologous reporter assay in E. coli [18], in which the $5^{\prime}$-UTR of all1871, from the TSS at position -137 with respect to the initiation codon (Mitschke et al., 2011), plus 60 nucleotides of the coding sequence of all1871 were fused to the sfgfp gene and co-expressed in E. coli with NsrR1 or with a control RNA. Accumulation of the GFP protein was measured in E. coli cells bearing different combinations of plasmids. The translation initiation region of all1871 was functional in E. coli resulting in significant GFP protein accumulation and GFP fluorescence (Figure 1B-D). The GFP fluorescence of cells bearing the all1871::sfgfp fusion (and the amount of GFP protein) decreased to less than $20 \%$ of the control when NsrR1 was co-expressed, indicating a direct interaction between NsrR1 and the $5^{\prime}$-UTR of all1871, which affects translation (Figure 1B-C).

To verify the interaction at the predicted site, a point mutation was introduced in NsrR1 affecting the predicted helix between NsrR1 and the 5'-UTR of all1871 (nucleotide 51, U to G). A compensatory 
mutation was introduced in the corresponding positions of the $5^{\prime}$-UTR of all1871 (nucleotide -13 with respect to the start codon, $\mathrm{G}$ to $\mathrm{C}$ ) and different combinations of the wild-type and mutated versions of both NsrR1 and 5'-UTR of all1871 were co-expressed. Mutation of nucleotide 51 in NsrR1 reduced the interaction between NsrR1 and the 5'-UTR of all1871, as suggested by the lower degree of fluorescence reduction with respect to the control (Figure 1C). When the mutated version of NsrR1 was combined with the mutated version of the mRNA containing the compensatory change, base pairing was restored resulting in a stronger fluorescence reduction than with wild-type NsrR1. Because of the long region of interaction, mutation of one single nucleotide produces a small effect, but differences observed are statistically significant. These data support a direct interaction of NsrR1 with the $5^{\prime}$-UTR of the all1871 mRNA that affects translation of All1871.

A

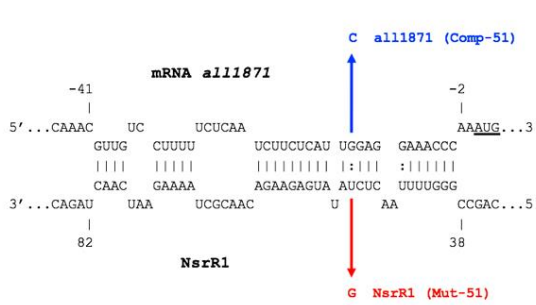

C
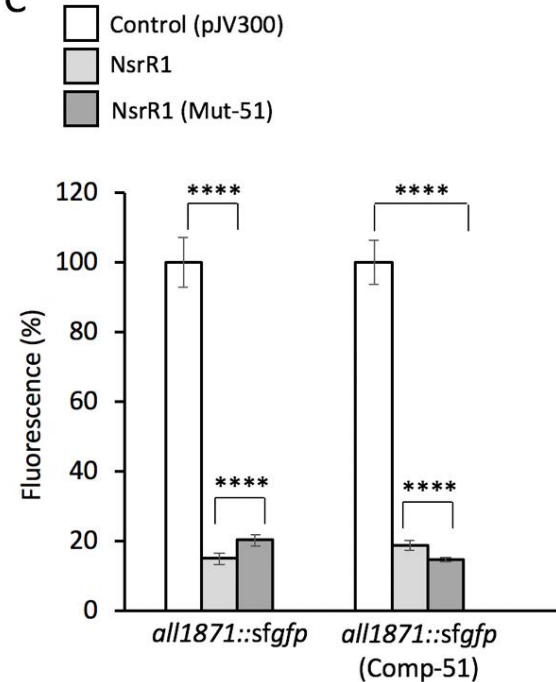

B

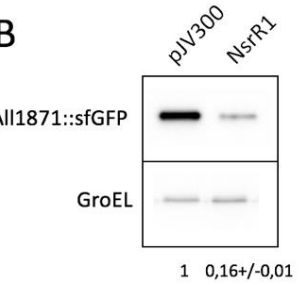

D

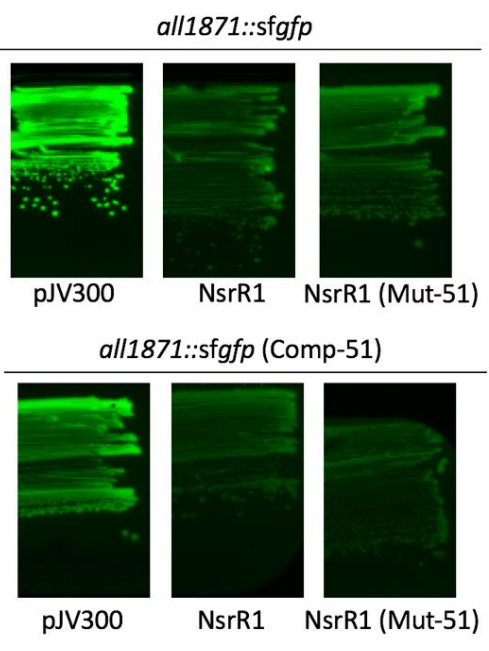

Figure 1. Verification of NsrR1 interaction with the $5^{\prime}$-UTR of all1871 using an in vivo reporter system. (A) Predicted interaction between NsrR1 and the 5'-UTR of all1871 according to IntaRNA [34]. all1871 nucleotides are numbered with respect to the start of the coding sequence (AUG start codon is underlined). The mutation introduced in NsrR1 at position 51 ( $\mathrm{U}$ to G, Mut-51) and the corresponding compensatory mutation in all1871 5' -UTR position -13 (G to C, Comp-51) are indicated in red and blue, respectively. (B) Accumulation of GFP protein in E. coli DH5 $\alpha$ cells bearing an all1871::sfgfp fusion combined with plasmid pJV300 (encoding a control RNA) or with a plasmid encoding NsrR1. Western blots were carried out using antibodies against GFP or GroEL. Numbers at the bottom of the image indicate relative GFP levels with respect to control after normalization with GroEL (average of two experiments). (C) Fluorescence measurements of E. coli DH5 $\alpha$ cultures bearing combinations of plasmids expressing different versions of NsrR1 (wild type or Mut-51) and all1871::sfofp fusions (wild type or Comp-51). Plasmid pJV300 (encoding a control RNA) was used as control. The data are presented as the mean $+/$ - standard deviation of cultures from eight independent colonies after subtraction of fluorescence in cells bearing $\mathrm{pXG0}$. Fluorescence is normalized to the $\mathrm{OD}_{600}$ of each culture. $T$-test $p$-value $<0.0001^{* * * *}$. (D) Fluorescence intensities of $E$ coli cells bearing all1871::sfgfp fusions (wild type or Comp-51) combined with different versions of NsrR1 (wild type or Mut-51) or with the control plasmid pJV300. Strains growing on LB agar plates were photographed under ultraviolet (UV) light. 
In addition, we have studied the interaction between NsrR1 and the all1871 mRNA by in vitro footprinting analysis. ${ }^{32} \mathrm{P}$-labelled NsrR1 was incubated with unlabeled all1871 mRNA (a fragment extending from positions -137 to +60 with respect to the start of the coding sequence) and probed with RNase T1, RNase A or lead(II) acetate (Figure 2A). A clear footprint was detected between positions 45 and 62 of NsrR1 (highlighted in the secondary structure model, Figure 2B), in good agreement with the bioinformatic prediction (Figure 1A). These in vitro results, therefore, confirm the in vivo results obtained from the analysis in E. coli using the sfGFP fusion system.

\section{A}

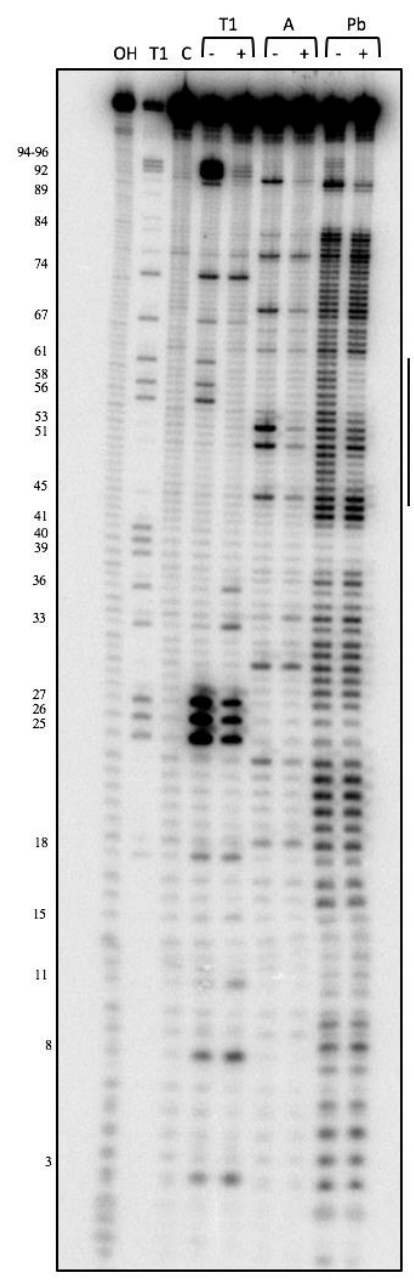

B

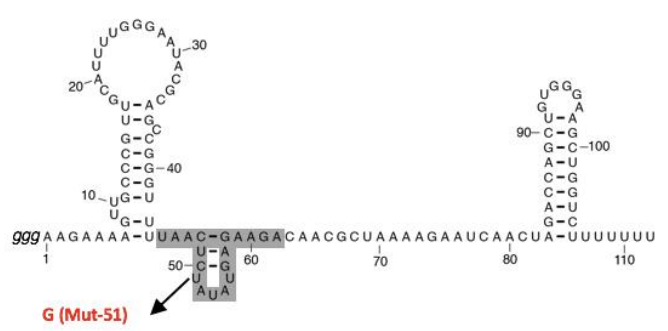

Figure 2. In vitro footprinting assay of the interaction between NsrR1 and all1871 5'-UTR. (A) RNase $\mathrm{T} 1$, RNase $\mathrm{A}$ or $\mathrm{Pb}(\mathrm{II})$ footprinting of the interaction between NsrR1 and the $5^{\prime}$-UTR of all1871. - /+ above lanes indicate absence/presence of all1871 5' UTR. The protected area is indicated by a vertical bar. NsrR1 was $5^{\prime}$ end-labelled. C, untreated control; OH, alkaline ladder; T1, RNase T1 ladder. Nucleotide positions of NsrR1 are shown on the left. (B) The nucleotides in NsrR1 involved in the interaction with the all1871 5'-UTR are indicated in grey on the previously described secondary structure model of NsrR1 [10]. The nucleotide changed ( $U$ to $G$ ) in version Mut-51 of NsrR1 is also indicated. 


\subsection{Expression of all1871 Is Regulated by Nitrogen Availability But Does Not Require NtcA or HetR}

According to global transcriptomic analyses, accumulation of the all1871 transcript is induced in response to nitrogen deficiency $[16,35]$. We have analyzed transcription from the TSS identified at position 2234072r [5] in the wild type strain and in two mutant derivatives, ntc $A$ mutant strain CSE2 [36] and hetR mutant strain DR884a [27]. Although in all strains there is significant expression in the presence of ammonium, transcription is similarly induced upon nitrogen step down in the wild type and in both mutant strains, suggesting the induction of transcription does not require NtcA or HetR (Figure 3). According to primer extension analysis, induction is transient both in the wild type and the $n t c A$ strain, with a decreased amount of transcript at $24 \mathrm{~h} v s .8 \mathrm{~h}$ after nitrogen removal, but in the het $R$ mutant the accumulation of transcript continues to be strong even at $24 \mathrm{~h}$ after nitrogen removal.
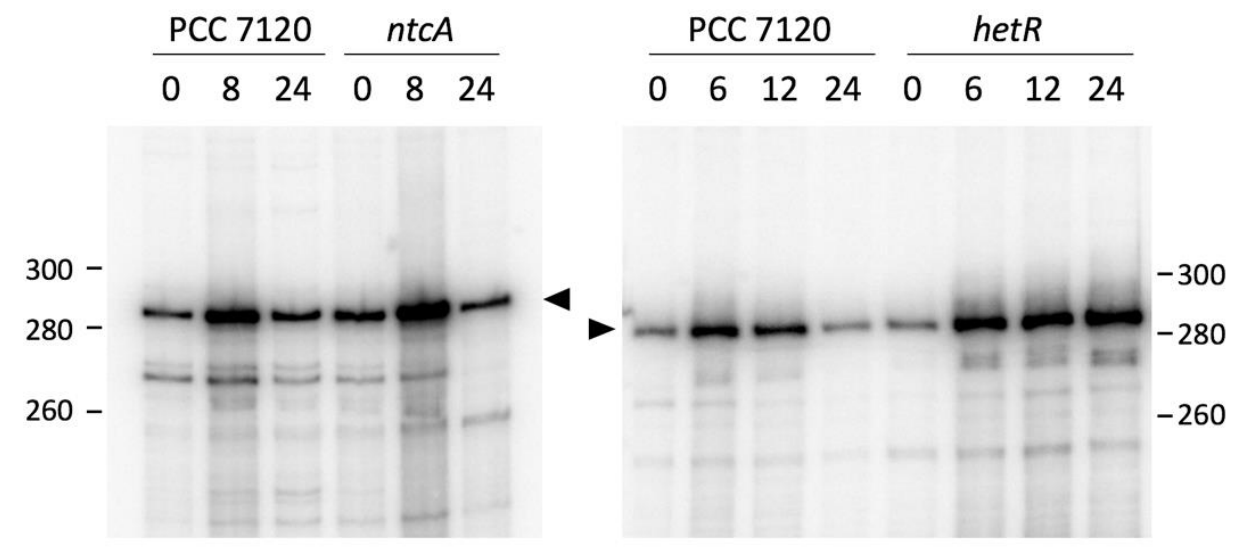

Figure 3. Nitrogen-regulated expression of all1871. Primer extension analysis of all1871 transcripts in Nostoc sp. PCC 7120 and its ntcA mutant derivative CSE2 (left panel) or in Nostoc sp. PCC 7120 and its hetR mutant derivative DR884a (right panel). Expression was analyzed in cells grown in the presence of ammonium and transferred to medium containing no source of combined nitrogen for the number of hours indicated. Size markers (nucleotides) are indicated. Triangles point to the products corresponding to the $5^{\prime}$ end at position 2234072r [5].

\subsection{Expression of all1871 Is Reduced by NsrR1 in Nostoc sp. PCC 7120}

In order to study in vivo in Nostoc the possible effect of NsrR1 on all1871 expression, we used a mutant strain that lacks NsrR1 ( $\Delta n s r R 1)$ [10]. Cells of strain $\Delta n s r R 1$ have no apparent difference in growth with respect to wild-type cells in media containing nitrate, ammonia, or lacking a source of combined nitrogen [10].

We have previously shown that transcription of NsrR1 is only partially (and transiently) repressed upon nitrogen step down, whereas maximal expression of NsrR1 is achieved upon ammonium addition to cells growing in the absence of combined nitrogen [10]. Therefore, to maximize the difference between wild-type cells expressing NsrR1 and cells lacking NsrR1 ( $\Delta n s r R 1$ strain), we chose to analyze cells grown in the absence of combined nitrogen $\left(\mathrm{N}_{2}\right)$ and compared them with cells grown in the absence of combined nitrogen to which ammonium was added and incubation continued for 4,8 or $24 \mathrm{~h}$ (Figure 4).

As previously described, expression of NsrR1 was induced and reached its highest level $8 \mathrm{~h}$ after ammonium addition (Figure 4A, middle panel). Concomitantly, expression of all1871 was repressed in the wild type, with a minimum in the sample corresponding to $8 \mathrm{~h}$ after ammonium addition (Figure 4A, upper panel). Furthermore, repression of all1871 was weaker in the strain lacking NsrR1, suggesting NsrR1 has a significant negative effect on the accumulation of all1871 mRNA in Nostoc, consistent with the interaction observed in the E. coli assay between NsrR1 and the all1871 mRNA. The effects of NsrR1 on the accumulation of All1871 protein were also analyzed by Western blot using antibodies we have generated against purified recombinant All1871 protein. The levels of All1871 
protein observed in extracts of strain $\triangle n s r R 1$ were about five-fold higher than those in the wild-type strain in the absence of combined nitrogen (Figure 4B). Consistent with the mRNA levels (Figure 4A), the reduction of the amount of All1871 protein in response to ammonium addition was stronger in the wild-type strain than in the $\Delta n s r R 1$ strain lacking NsrR1.
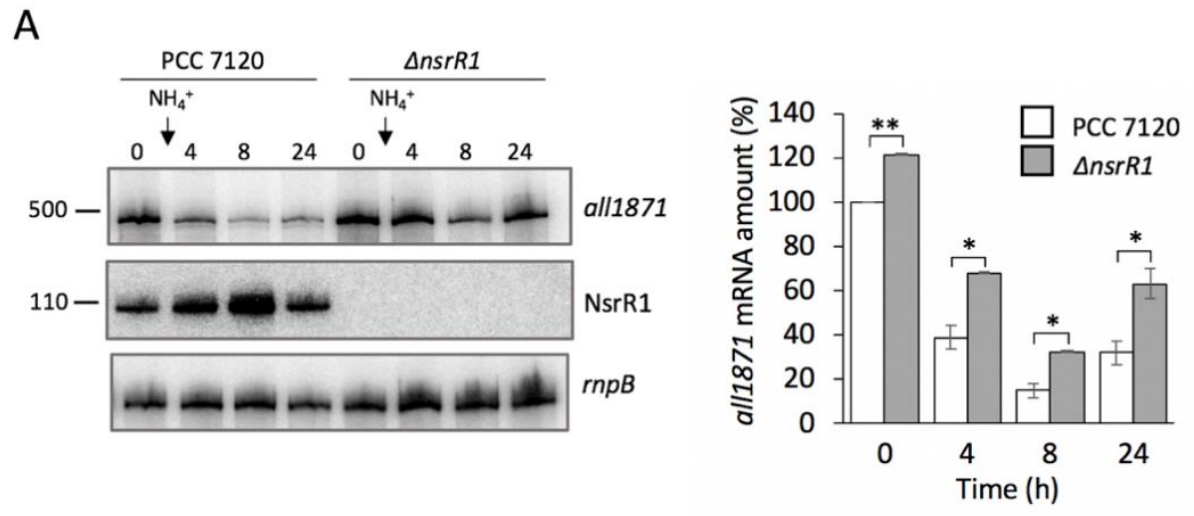

B

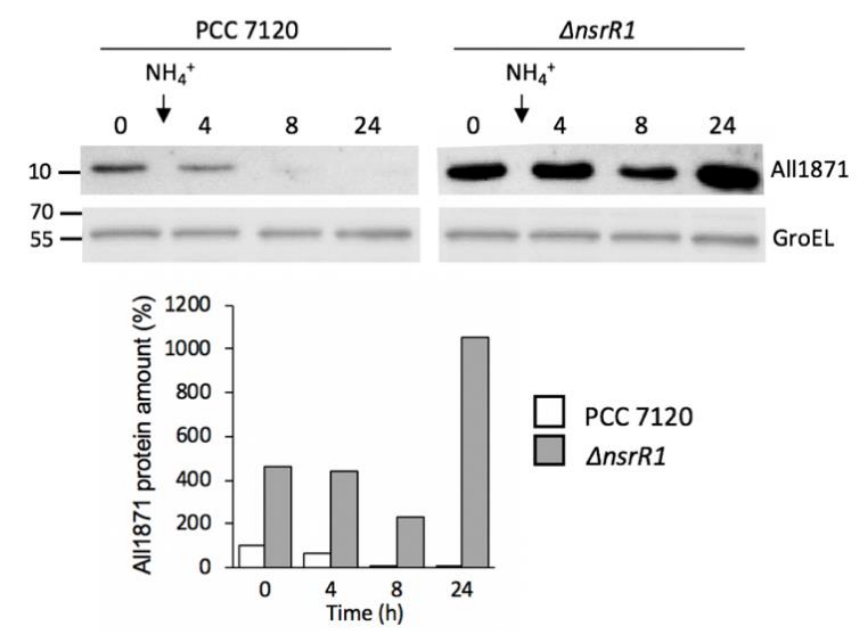

Figure 4. NsrR1 affects nitrogen-regulated expression of all1871. (A) Expression of all1871 was analyzed by Northern blot in Nostoc sp. PCC 7120 and in a mutant strain lacking NsrR1 ( $\Delta$ nsrR1). Cells were grown in nitrogen-free liquid medium for one week (steady-state $\mathrm{N}_{2}$-fixing cultures) and $10 \mathrm{mM}$ ammonium chloride was added to increase expression of NsrR1. Samples were taken before ammonium addition ( 0 ) and at the number of hours indicated after ammonium addition. The upper panel shows hybridization to the all1871 probe. The middle panel shows hybridization to the probe for NsrR1. The lower panel shows hybridization to a probe for $r п p B$ used as loading and transfer control. Quantification of all1871 mRNA accumulation is indicated on the right, relative to the amount present in the wild type strain in $\mathrm{N}_{2}$ using the amount of $r n p B$ for normalization. Results from two technical replicates were averaged. $T$-test $p$-value $<0.05^{*} ;<0.01^{* *}$. (B) Accumulation of the All1871 protein was determined by Western blot in samples containing $40 \mu \mathrm{g}$ of soluble fraction from cells analyzed in (A). Upper panels show detection of All1871. Lower panels show detection of GroEL, used as loading and transfer control. Quantification of All1871 protein is shown at the bottom, relative to the amount present in the wild type strain in $\mathrm{N}_{2}$. Quantification was performed with ImageLab software (Bio-Rad) using the amount of GroEL for normalization. One representative experiment is shown.

\subsection{All1871 Is Differentially Expressed in Heterocysts and Required for Diazotrophic Growth But Not for} Heterocyst Differentiation

In order to obtain an insight into a possible function of All1871, we analyzed expression of all1871 along filaments of Nostoc. We prepared plasmid pELV75 containing a segment from position -200 
with respect to the TSS of all1871 plus a translational fusion between the entire all1871 gene and the gfpmut2 gene. This plasmid was introduced in Nostoc by conjugation. Integration of the plasmid in the region encoding all1871 leads to a partial duplication of this region in which the translational fusion is preceded by the natural context of all1871 in the wild type strain (Figure 5A).
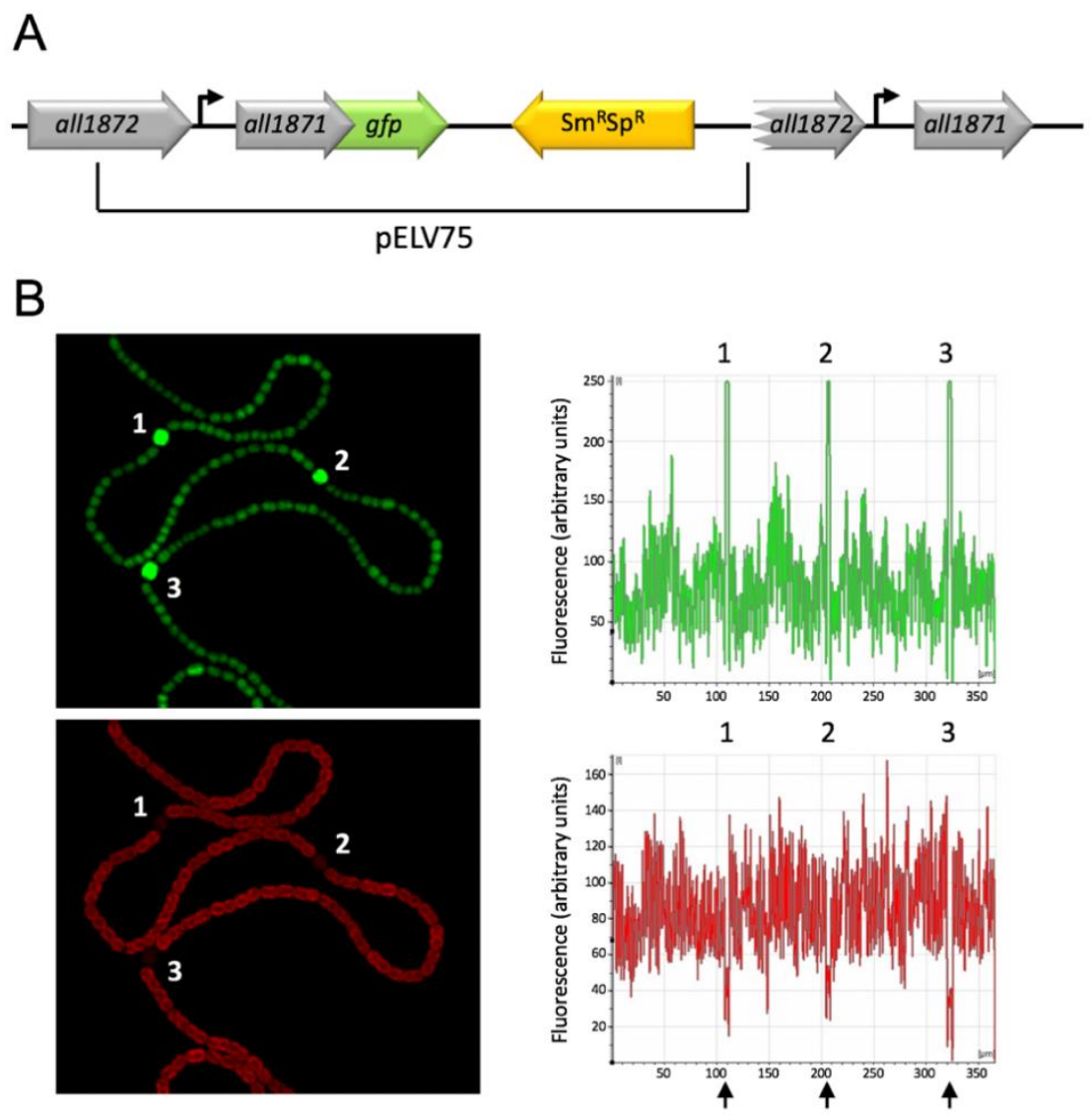

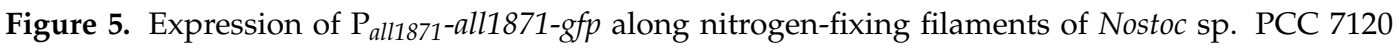
bearing pELV75. (A) Schematic representation of plasmid pELV75 integrated by single recombination in the chromosomal region encoding all1871. The location of the transcriptional start site of all1871 (bent arrows) and the segment (from -336 with respect to the start codon of all1871) fused to $g f p$ in plasmid pELV75 are depicted. Not drawn to scale. (B) Confocal fluorescence image of a filament growing on top of nitrogen-free medium is shown for the green channel (upper panel, GFP fluorescence) and red channel (lower panel, autofluorescence). Quantification of the green and red signals along the filament is shown on the right of each image. Mature heterocysts are indicated with numbers. The positions of lowest autofluorescence, corresponding to heterocysts, are indicated with black arrows.

GFP fluorescence was analyzed by confocal fluorescence microscopy of filaments growing on top of medium lacking combined nitrogen (Figure 5B). Quantification of the green signal (GPF) and the red autofluorescence along the filaments showed that although all cells showed green fluorescence, fluorescence peaks were associated with cells that had differentiated as heterocysts, as indicated by their larger size and reduced red autofluorescence.

We then prepared an all1871 null mutant by interrupting the all1871 gene with a $\mathrm{Nm}^{\mathrm{R}}$ gene (Figure S2A). Complete segregation of mutant chromosomes was verified by PCR amplification and Western blot with antibodies against All1871 (Figure S2B-C). Cells of the all1871::Nm mutant were unable to grow on plates in the absence of combined nitrogen but showed no growth defect in the presence of combined nitrogen (nitrate) (Figure 6A). The mutation was complemented by introduction of a plasmid bearing all1871 expressed from the trc promoter from E. coli, that provides constitutive 
expression in Nostoc (see e.g., [37]), leading to partial recovery of the wild-type phenotype, as indicated by the greenish growth of complemented cells vs. the blue-green growth of wild-type cells (Figure 6A).

A

$\mathrm{NO}_{3}^{-}$

PCC 7120
all1871::Nm
all1871::Nm + $P_{\text {trc }}:$ all1871
PCC 7120
all1871::Nm
all1871:: Nm + $P_{\text {trc }}:$ all1871

B

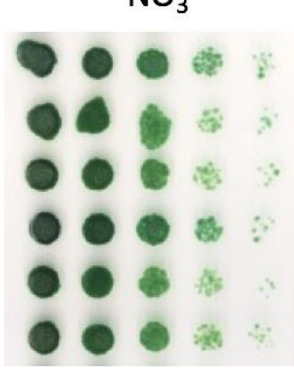

$\mathrm{N}_{2}$

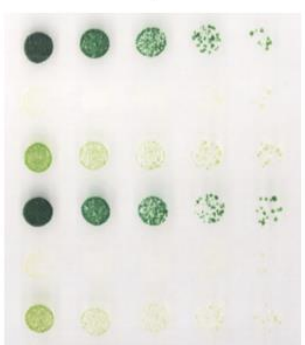

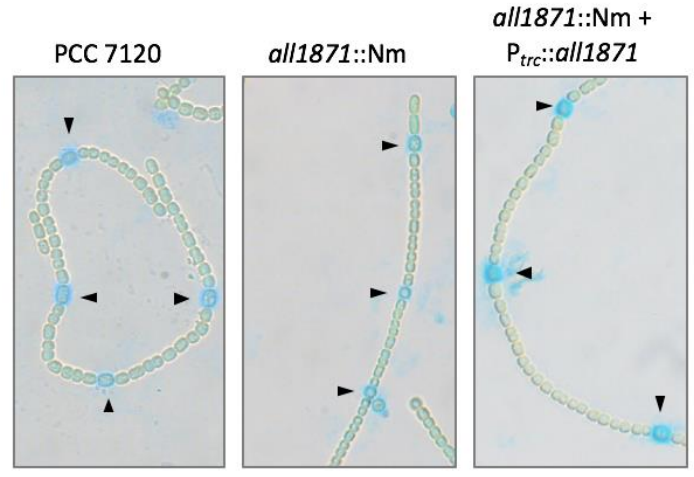

C

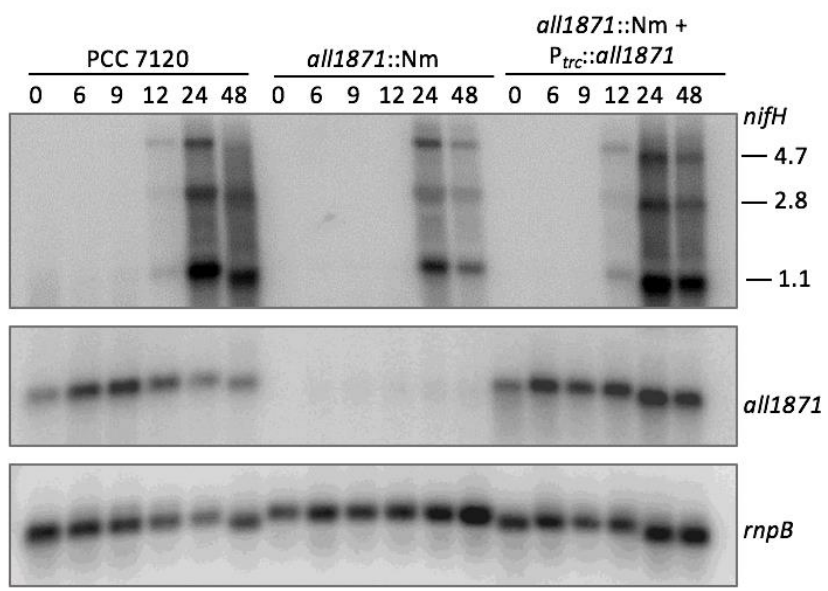

Figure 6. all1871 mutants are defective in diazotrophic growth. (A) Cells were grown in the presence of nitrate, washed and resuspended in $\mathrm{BG}_{1} 1_{0}$ at an $\mathrm{OD}_{750}=0.3$. Five-fold serial dilutions of liquid cultures of wild type, insertional mutant all1871::Nm and complemented mutant strain (all1871::Nm + $P_{t r c}:$ all1871) were prepared and $10 \mu \mathrm{L}$ of each dilution plated on BG11 0 plates lacking nitrogen $\left(\mathrm{N}_{2}\right)$ or containing nitrate $\left(\mathrm{NO}_{3}{ }^{-}\right)$. Two different clones of each strain were analyzed. Pictures were taken after 10 days $\left(\mathrm{NO}_{3}{ }^{-}\right)$or 13 days $\left(\mathrm{N}_{2}\right)$ of incubation at $30{ }^{\circ} \mathrm{C}$. (B) Alcian blue staining of heterocyst polysaccharides in filaments of Nostoc sp. PCC 7120, insertional mutant all1871::Nm and complemented mutant strain (all1871::Nm + $\mathrm{P}_{\text {trc }}:$ all1871), $26 \mathrm{~h}$ after combined nitrogen removal. (C) Northern blots with RNA isolated from the indicated strains at different time points (indicated in hours) after nitrogen removal and hybridized with probes for $n i f H$ (upper panel), all1871 (middle panel) and $r n p B$ (lower panel) as loading control. Sizes of the nifH transcripts are indicated on the right in kb. 
We wondered whether the observed defects in diazotrophic growth of the all1871::Nm strain were due to lack of heterocyst differentiation or heterocyst function. Because steady-state nitrogen-fixing cultures could not be obtained, we analyzed morphological differentiation of heterocysts $26 \mathrm{~h}$ after combined nitrogen removal by staining the cells with Alcian blue, a molecule that binds to the external polysaccharide layer of heterocysts. Figure $6 \mathrm{~B}$ shows the presence of Alcian blue-stained cells in a regular pattern similar to the wild type in the mutant strain all1871::Nm as well as in the complemented strain all1871::Nm $+\mathrm{P}_{\text {trc }}$-all1871, indicating differentiation of heterocysts took place in both strains, at least to the relatively initial stage in which polysaccharides are deposited outside the outer membrane of the vegetative cells undergoing differentiation into heterocysts. As an indication of heterocyst maturity, transcription of the nifHDK genes encoding nitrogenase was also analyzed by Northern blot (Figure 6C). Transcription of nifHDK seems delayed and reduced in the all1871::Nm mutant. Again, complementation with a plasmid bearing all1871 expressed from the trc promoter from E. coli leads to recovery of the wild-type timing of nifHDK expression.

Finally, we have measured the nitrogenase activity of the different strains (Table 1). Strain all1871::Nm had no detectable nitrogenase activity in either oxic or anoxic conditions. This result excludes the possibility that the absence of activity in strain all1871::Nm was due to a defect in the maturation of heterocyst envelopes that results in the presence of inactivating $\mathrm{O}_{2}$ amounts inside the heterocyst.

Table 1. Nitrogenase activity of different Nostoc strains.

\begin{tabular}{|c|c|c|}
\hline \multirow{2}{*}{ Strain } & \multicolumn{2}{|c|}{ Nitrogenase Activity ${ }^{1}\left(\mu \mathrm{mol}\right.$ Ethylene $\left.\cdot \mathrm{h}^{-1} \cdot \mathrm{mg} \mathrm{Chl}^{-1}\right)$} \\
\hline & Oxic Conditions & Anoxic Conditions \\
\hline Nostoc sp. PCC 7120 & $10.50 \pm 3.49$ & $10.64 \pm 2.05$ \\
\hline all1871::Nm & $0.00 \pm 0.00$ & $0.00 \pm 0.00$ \\
\hline $\begin{array}{c}\text { all1871::Nm + } \\
\mathrm{P}_{\text {trc }}:: \text { all1871 }\end{array}$ & $3.66 \pm 1.67$ & $5.05 \pm 3.41$ \\
\hline
\end{tabular}

\footnotetext{
${ }^{1}$ Nitrogenase activity was measured in cultures grown with nitrate and transferred for $24 \mathrm{~h}$ to nitrogen-free medium. Data are the average and standard deviation of assays performed with two independent cultures of Nostoc sp. PCC 7120 and strain all1871::Nm $+P_{\text {trc }}:$ all1871 or eight independent cultures of strain all1871:::Nm.
}

\section{Discussion}

Small RNAs are important components in regulatory networks that involve classical transcription factors. For instance, in E. coli, members of the CRP/FNR family of transcriptional regulators are known to control the expression of several small RNAs, all of them contributing to the regulatory effects exerted by these two major transcription factors [2]. Cyanobacterial transcription factors also regulate the expression of small RNAs that exert regulatory functions involved in the adaptation to different environmental situations [38]. For instance, the transcriptional regulator RpaB and small RNA PsrR1 constitute a feed-forward loop controlling acclimation to different light intensities [39]. Accumulating evidence concerning nitrogen-regulated non-coding RNAs indicates this type of molecules is involved in NtcA-mediated post-transcriptional regulation [5,9]. One case analyzed in detail is NsiR4, whose transcription is induced in response to nitrogen deficiency and is involved in post-transcriptional regulation of glutamine synthetase [8]. Expression of another sRNA, NsrR1, is repressed by NtcA in response to nitrogen deficiency. Among predicted targets of NsrR1 are nblA [10], and all1871, whose interaction with NsrR1 we describe in this work.

Interaction between homologs of NsrR1 and homologs of all1871 is conserved in many cyanobacteria encoding NsrR1 (Figure S1), consistent with the observation that all1871 has the highest score in CopraRNA predictions of candidates to be regulated by NsrR1 [10]. Using an in vivo reporter system established in E. coli we demonstrate that NsrR1 represses translation, leading to reduced expression of an all1871::sfGFP fusion in the presence of NsrR1. A direct interaction of NsrR1 
with the predicted region in the $5^{\prime}$-UTR of all1871 was also verified by a point mutation in the region involved (Figure 1) and by in vitro footprinting experiments (Figure 2).

We have demonstrated higher accumulation of all1871 transcripts in a strain of Nostoc sp. PCC 7120 that lacks NsrR1 ( $\Delta n s r R 1)$ than in wild-type cells cultured in the absence of combined nitrogen (Figure 4A). Upon addition of ammonium, that induces NsrR1 expression, the amount of all1871 mRNA is strongly reduced in the wild type but not in the $\Delta n s r R 1$ strain. Similarly, the amount of All1871 protein, detected with a specific antibody (Figure 4B), is much higher in cells lacking NsrR1 than in the wild-type strain. Upon the addition of ammonium, the amount of All1871 protein is reduced in the wild type, becoming barely detectable $8 \mathrm{~h}$ after ammonium addition, but only slightly reduced in the $\Delta n s r R 1$ strain. The magnitude of the repression upon ammonium addition is higher at the protein level than at the transcript level, in agreement with NsrR1 inhibiting translation through its interaction with the $5^{\prime}$-UTR of all1871. The reduced accumulation of all1871 mRNA in the presence of NsrR1 can be attributed to destabilization of the mRNA when translation is inhibited.

Upregulation of all1871 upon nitrogen deprivation would be mediated, at least in part, by alleviating the repression exerted by NsrR1, whose transcription is repressed upon nitrogen stress. NsrR1 repression is only partially dependent on NtcA, with an additional factor possibly involved [10], explaining that up-regulation of all1871 is independent of NtcA (Figure 3). The observation that there is partial repression of all1871 expression upon ammonium addition even in the $\Delta n s r R 1$ mutant strain (Figure 4) points to some additional factor(s) being involved in regulation of all1871. In any case, because the interaction between NsrR1 and all1871 takes place at the translation initiation region, even if another regulatory mechanism influences transcription of all1871, the accumulation of the All1871 protein would be ultimately regulated by nitrogen availability through NsrR1 (Figure 7).

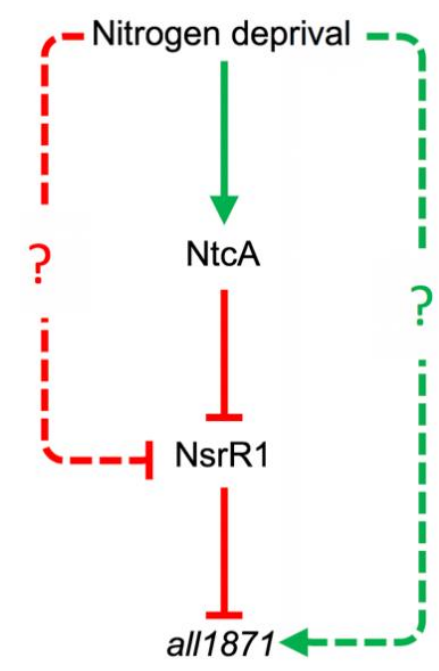

Figure 7. Schematic representation of elements involved in the regulation of expression of all1871. Green arrows represent positive effects. Red arrows represent negative effects. Dashed lines represent mechanisms operated by currently unknown factors (question marks).

By means of a translational fusion to the $g f p$ gene we have shown higher expression of all1871 in heterocysts than in vegetative cells (Figure 5). We have also shown that expression of all1871 does not require HetR (Figure 3) suggesting it is not heterocyst-specific. Taken together these observations point to the operation of post-transcriptional regulatory mechanisms leading to differential accumulation of all1871 transcripts in heterocysts vs. vegetative cells. Because NtcA is subjected to differential accumulation in heterocysts [31,40], the amount of NsrR1 is also expected to be different. A higher amount of NtcA in heterocysts would result in lower levels of NsrR1, and consequently increased translation of All1871 in these specialized cells. NsrR1 would thus contribute to differential expression in heterocysts of a transcript found to be required for diazotrophic growth. Whether the stronger accumulation of All1871-GFP protein in heterocysts (Figure 5) is only a consequence of 
reduced post-transcriptional regulation by NsrR1 or involves additional differential regulation in these specialized cells, is currently unknown.

All1871 is conserved in many unicellular and filamentous strains of cyanobacteria. Its function is unknown and it does not contain domains of known function. Strong accumulation in heterocysts together with the observation that its expression is regulated by NsrR1, a nitrogen-repressed sRNA, suggest a possible role in the response to nitrogen stress and/or in heterocyst function. A mutant lacking All1871 (all1871::Nm) could not grow in media lacking combined nitrogen (Figure 6A). The inability to grow in media lacking combined nitrogen of the all1871::Nm strain is only partially complemented by constitutive expression of all1871 from $\mathrm{P}_{\text {trc }}$ (Figure 6A). Such phenotype could be explained by toxicity due to unregulated expression of all1871 in this strain, in contrast to the transient induction observed in the wild type upon nitrogen step-down. It can also be speculated that constitutive expression of all1871 in the complemented strain could lead to sequestration of NsrR1 affecting accumulation of other mRNAs also regulated by NsrR1, such as nblA [10]. However, it has been previously shown that cells lacking NsrR1 have no apparent phenotypic differences with respect to wild-type cells [10]. Despite its ability to develop Alcian blue-stained cells (Figure 6B), suggestive of heterocyst differentiation, and the induction of nitrogenase transcription (Figure 6C), all1871::Nm filaments lacked nitrogenase activity (Table 1), indicating that All1871 is required for heterocyst function through currently unknown mechanisms. We have observed that in the absence of combined nitrogen, filaments of the all1871::Nm strain are extensively fragmented in liquid media (not shown). Because exchange of metabolites between vegetative cells and heterocysts is a requisite for sustained nitrogen fixation, filament fragmentation would preclude diazotrophic growth of the all1871::Nm strain.

In summary, in this work we describe a new gene required for diazotrophic growth, although not for heterocyst differentiation, and characterize its regulation by NsrR1, a nitrogen-regulated small RNA involved in acclimation to nitrogen deficiency.

Supplementary Materials: The following are available online at http://www.mdpi.com/2075-1729/10/5/54/s1: Figure S1: Conservation in cyanobacteria of the predicted interaction between the mRNA of all1871 and NsrR1; Figure S2: Construction of mutant all1871::Nm; Table S1: Strains; Table S2: Oligonucleotides; Table S3: Plasmids; Table S4: Sequences of inserts in plasmids containing NsrR1 used for verification in E. coli; Table S5: Sequences of inserts in the all1871-sfgfp fusion plasmids; Table S6: Sequences of templates used for in vitro transcription.

Author Contributions: Conceptualization, A.V. and A.M.M.-P.; Formal analysis, A.V. and A.M.M.-P.; Funding acquisition, A.M.M.-P.; Investigation, I.Á.-E., M.B.-Á., E.O.-V., A.V. and A.M.M.-P.; Methodology, A.V. and A.M.M.-P.; Supervision, A.V. and A.M.M.-P.; Writing—original draft, A.M.M.-P.; Writing-review and editing, I.Á.-E., M.B.-Á., A.V., E.O.-V. and A.M.M.-P. All authors have read and agreed to the published version of the manuscript.

Funding: This research was funded by Ministerio de Economía y Competitividad, grant number BFU2013-48282C2-1-P, and by Agencia Estatal de Investigación (AEI), Ministerio de Economía, Industria y Competitividad, grant number BFU2016-74943-C2-1-P, both cofinanced by Fondo Europeo de Desarrollo Regional (FEDER). IA-E is the recipient of a predoctoral contract from Ministerio de Economía y Competitividad, Spain (BES-2014-068488). MB-A is the recipient of a predoctoral contract from Ministerio de Educación, Cultura y Deporte, Spain (FPU014/05123 and EST16-00088).

Acknowledgments: We thank M. Isabel Muro-Pastor (IBVF, CSIC-Universidad de Sevilla) for help with protein purification, and Alicia Orea (IBVF, CSIC- Universidad de Sevilla) for technical assistance with microscopy.

Conflicts of Interest: The authors declare no conflict of interest.

\section{References}

1. Wagner, E.G.; Romby, P. Small RNAs in bacteria and archaea: Who they are, what they do, and how they do it. Adv. Genet. 2015, 90, 133-208. [CrossRef]

2. Brosse, A.; Guillier, M. Bacterial small RNAs in mixed regulatory networks. Microbiol. Spectr. 2018, 6, RWR-0014-2017. [CrossRef]

3. Nitzan, M.; Rehani, R.; Margalit, H. Integration of bacterial small RNAs in regulatory networks. Annu. Rev. Biophys. 2017, 46, 131-148. [CrossRef] 
4. Herrero, A.; Muro-Pastor, A.M.; Flores, E. Nitrogen control in cyanobacteria. J. Bacteriol. 2001, 183, 411-425. [CrossRef]

5. Mitschke, J.; Vioque, A.; Haas, F.; Hess, W.R.; Muro-Pastor, A.M. Dynamics of transcriptional start site selection during nitrogen stress-induced cell differentiation in Anabaena sp. PCC7120. Proc. Natl. Acad. Sci. USA 2011, 108, 20130-20135. [CrossRef]

6. Picossi, S.; Flores, E.; Herrero, A. ChIP analysis unravels an exceptionally wide distribution of DNA binding sites for the NtcA transcription factor in a heterocyst-forming cyanobacterium. BMC Genom. 2014, 15, 22. [CrossRef]

7. Giner-Lamia, J.; Robles-Rengel, R.; Hernández-Prieto, M.A.; Muro-Pastor, M.I.; Florencio, F.J.; Futschik, M.E. Identification of the direct regulon of NtcA during early acclimation to nitrogen starvation in the cyanobacterium Synechocystis sp. PCC 6803. Nucleic Acids Res. 2017, 45, 11800-11820. [CrossRef]

8. Klähn, S.; Schaal, C.; Georg, J.; Baumgartner, D.; Knippen, G.; Hagemann, M.; Muro-Pastor, A.M.; Hess, W.R. The sRNA NsiR4 is involved in nitrogen assimilation control in cyanobacteria by targeting glutamine synthetase inactivating factor IF7. Proc. Natl. Acad. Sci. USA 2015, 112, E6243-E6252. [CrossRef]

9. Brenes-Álvarez, M.; Olmedo-Verd, E.; Vioque, A.; Muro-Pastor, A.M. Identification of conserved and potentially regulatory small RNAs in heterocystous cyanobacteria. Front. Microbiol. 2016, 7, 48. [CrossRef]

10. Álvarez-Escribano, I.; Vioque, A.; Muro-Pastor, A.M. NsrR1, a nitrogen stress-repressed sRNA, contributes to the regulation of $n b l A$ in Nostoc sp. PCC 7120. Front. Microbiol. 2018, 9, 2267. [CrossRef]

11. Collier, J.L.; Grossman, A.R. A small polypeptide triggers complete degradation of light-harvesting phycobiliproteins in nutrient-deprived cyanobacteria. EMBO J. 1994, 13, 1039-1047. [CrossRef]

12. García-Domínguez, M.; Reyes, J.C.; Florencio, F.J. NtcA represses transcription of gifA and gifB, genes that encode inhibitors of glutamine synthetase type I from Synechocystis sp. PCC 6803. Mol. Microbiol. 2000, 35, 1192-1201. [CrossRef]

13. Flores, E.; Herrero, A. Compartmentalized function through cell differentiation in filamentous cyanobacteria. Nature Rev. Microbiol. 2010, 8, 39-50. [CrossRef]

14. Muro-Pastor, A.M.; Hess, W.R. Heterocyst differentiation: From single mutants to global approaches. Trends Microbiol. 2012, 20, 548-557. [CrossRef]

15. Olmedo-Verd, E.; Brenes-Álvarez, M.; Vioque, A.; Muro-Pastor, A.M. A heterocyst-specific antisense RNA contributes to metabolic reprogramming in Nostoc sp. PCC 7120. Plant Cell Physiol. 2019, 60, 1646-1655. [CrossRef]

16. Brenes-Álvarez, M.; Mitschke, J.; Olmedo-Verd, E.; Georg, J.; Hess, W.R.; Vioque, A.; Muro-Pastor, A.M. Elements of the heterocyst-specific transcriptome unravelled by co-expression analysis in Nostoc sp. PCC 7120. Environ. Microbiol. 2019, 21, 2544-2558. [CrossRef]

17. Rippka, R.; Deruelles, J.; Waterbury, J.B.; Herdman, M.; Stanier, R.Y. Generic assignments, strain stories and properties of pure cultures of cyanobacteria. J. Gen. Microbiol. 1979, 111,1-61.

18. Urban, J.H.; Vogel, J. Translational control and target recognition by Escherichia coli small RNAs in vivo. Nucleic Acids Res. 2007, 35, 1018-1037. [CrossRef]

19. Corcoran, C.P.; Podkaminski, D.; Papenfort, K.; Urban, J.H.; Hinton, J.C.; Vogel, J. Superfolder GFP reporters validate diverse new mRNA targets of the classic porin regulator, MicF RNA. Mol. Microbiol. 2012, 84, 428-445. [CrossRef]

20. Sittka, A.; Pfeiffer, V.; Tedin, K.; Vogel, J. The RNA chaperone Hfq is essential for the virulence of Salmonella typhimurium. Mol. Microbiol. 2007, 63, 193-217. [CrossRef]

21. Wright, P.R.; Richter, A.S.; Papenfort, K.; Mann, M.; Vogel, J.; Hess, W.R.; Backofen, R.; Georg, J. Comparative genomics boosts target prediction for bacterial small RNAs. Proc. Natl. Acad. Sci. USA 2013, 110, E3487-E3496. [CrossRef]

22. Mohamed, A.; Jansson, C. Influence of light on accumulation of photosynthesis-specific transcripts in the cyanobacterium Synechocystis 6803. Plant Mol. Biol. 1989, 13, 693-700. [CrossRef]

23. Muro-Pastor, A.M.; Valladares, A.; Flores, E.; Herrero, A. The hetC gene is a direct target of the NtcA transcriptional regulator in cyanobacterial heterocyst development. J. Bacteriol. 1999, 181, 6664-6669. [CrossRef]

24. Steglich, C.; Futschik, M.E.; Lindell, D.; Vo $\beta$, B.; Chisholm, S.W.; Hess, W.R. The challenge of regulation in a minimal photoautotroph: Non-coding RNAs in Prochlorococcus. PLoS Genet. 2008, 4, e1000173. [CrossRef] 
25. Vioque, A. Analysis of the gene encoding the RNA subunit of ribonuclease P from cyanobacteria. Nucleic Acids Res. 1992, 20, 6331-6337. [CrossRef]

26. Merino-Puerto, V.; Mariscal, V.; Mullineaux, C.W.; Herrero, A.; Flores, E. Fra proteins influencing filament integrity, diazotrophy and localization of septal protein SepJ in the heterocyst-forming cyanobacterium Anabaena sp. Mol. Microbiol. 2010, 75, 1159-1170. [CrossRef]

27. Black, T.A.; Cai, Y.; Wolk, C.P. Spatial expression and autoregulation of hetR, a gene involved in the control of heterocyst development in Anabaena. Mol. Microbiol. 1993, 9, 77-84. [CrossRef]

28. Elhai, J.; Wolk, C.P. Conjugal transfer of DNA to cyanobacteria. Methods Enzymol. 1988, 167, 747-754.

29. Cai, Y.; Wolk, C.P. Use of a conditionally lethal gene in Anabaena sp. strain PCC 7120 to select for double recombinants and to entrap insertion sequences. J. Bacteriol. 1990, 172, 3138-3145. [CrossRef]

30. Muro-Pastor, A.M.; Flores, E.; Herrero, A. NtcA-regulated heterocyst differentiation genes hetC and $\operatorname{dev} B$ from Anabaena sp. strain PCC 7120 exhibit a similar tandem promoter arrangement. J. Bacteriol. 2009, 191, 5765-5774. [CrossRef]

31. Olmedo-Verd, E.; Muro-Pastor, A.M.; Flores, E.; Herrero, A. Localized induction of the ntcA regulatory gene in developing heterocysts of Anabaena sp. strain PCC 7120. J. Bacteriol. 2006, 188, 6694-6699. [CrossRef]

32. Muro-Pastor, A.M. The heterocyst-specific NsiR1 small RNA is an early marker of cell differentiation in cyanobacterial filaments. mBio 2014, 5, e01079-14. [CrossRef]

33. Olmedo-Verd, E.; Flores, E.; Herrero, A.; Muro-Pastor, A.M. HetR-dependent and -independent expression of heterocyst-related genes in an Anabaena strain overproducing the NtcA transcription factor. J. Bacteriol. 2005, 187, 1985-1991. [CrossRef]

34. Mann, M.; Wright, P.R.; Backofen, R. IntaRNA 2.0: Enhanced and customizable prediction of RNA-RNA interactions. Nucleic Acids Res. 2017, 45, W435-W439. [CrossRef]

35. Flaherty, B.L.; Van Nieuwerburgh, F.; Head, S.R.; Golden, J.W. Directional RNA deep sequencing sheds new light on the transcriptional response of Anabaena sp. strain PCC 7120 to combined-nitrogen deprivation. BMC Genom. 2011, 12, 332. [CrossRef]

36. Frías, J.E.; Flores, E.; Herrero, A. Requirement of the regulatory protein NtcA for the expression of nitrogen assimilation and heterocyst development genes in the cyanobacterium Anabaena sp. PCC 7120. Mol. Microbiol. 1994, 14, 823-832. [CrossRef]

37. Brenes-Álvarez, M.; Vioque, A.; Muro-Pastor, A.M. The integrity of the cell wall and its remodeling during heterocyst differentiation are regulated by phylogenetically conserved small RNA Yfr1 in Nostoc sp. strain PCC 7120. mBio 2020, 11, e02599-19. [CrossRef]

38. Muro-Pastor, A.M.; Hess, W.R. Regulatory RNA at the crossroads of carbon and nitrogen metabolism in photosynthetic cyanobacteria. Biochim. Biophys. Acta Gene Regul. Mech. 2020, 1863, 194477. [CrossRef]

39. Kadowaki, T.; Nagayama, R.; Georg, J.; Nishiyama, Y.; Wilde, A.; Hess, W.R.; Hihara, Y. A feed-forward loop consisting of the response regulator RpaB and the small RNA PsrR1 controls light acclimation of photosystem I gene expression in the cyanobacterium Synechocystis sp. PCC 6803. Plant Cell Physiol. 2016, 57, 813-823. [CrossRef]

40. Sandh, G.; Ramstrom, M.; Stensjö, K. Analysis of the early heterocyst Cys-proteome in the multicellular cyanobacterium Nostoc punctiforme reveals novel insights into the division of labor within diazotrophic filaments. BMC Genom. 2014, 15, 1064. [CrossRef]

(C) 2020 by the authors. Licensee MDPI, Basel, Switzerland. This article is an open access article distributed under the terms and conditions of the Creative Commons Attribution (CC BY) license (http://creativecommons.org/licenses/by/4.0/). 